livraisons

d'Histoire

de l'Architecture

\section{Livraisons de l'histoire de l'architecture}

34 | 2017

Le bois

\title{
L'activité d'un architecte diocésain : Pierre Félix Delarue au Mans : la restauration du porche méridional de la cathédrale (1837-1841)
}

The activity of a diocesan architect

Die Tätigkeit eines Diözesanarchitekten : Pierre-Félix Delarue in Le Mans : die

Restaurierung des Südportales der Kathedrale (1837-1841)

Delphine Goutierre

\section{OpenEdition}

Journals

Édition électronique

URL : http://journals.openedition.org/lha/864

DOI : $10.4000 /$ Iha. 864

ISSN : 1960-5994

Éditeur

Association Livraisons d'histoire de l'architecture - LHA

Édition imprimée

Date de publication : 15 décembre 2017

Pagination : 81-92

ISSN : $1627-4970$

\section{Référence électronique}

Delphine Goutierre, «L'activité d'un architecte diocésain : Pierre Félix Delarue au Mans : la restauration du porche méridional de la cathédrale (1837-1841) », Livraisons de l'histoire de l'architecture [En ligne], 34 | 2017, mis en ligne le 15 décembre 2019, consulté le 22 mars 2020. URL : http:// journals.openedition.org/lha/864; DOI : https://doi.org/10.4000/lha.864 
Varia 



\section{L'ACTIVITÉ D'UN ARCHITECTE DIOCÉSAIN : PIERRE-FÉLIX DELARUE AU MANS : LA RESTAURATION DU PORCHE MÉRIDIONAL DE LA CATHÉDRALE (1837-1841)}

Pierre-Félix Delarue (1795-1873) fut architecte départemental de la Sarthe de 1824 à 1864. Au rang de ses réalisations les plus connues figure l'ancien asile d'aliénés manceau de la rue Étoc Demazy, l'un des premiers établissements du genre édifiés en France durant la première moitié du $\mathrm{XIX}^{\mathrm{e}}$ siècle ${ }^{1}$ et classé au titre des monuments historiques le 4 octobre $2001^{2}$. Delarue fut également architecte diocésain de ce même département jusqu'en 1849, quelque temps après que l'administration des Cultes eût créé ce corps en $1848^{3}$. À ce titre il fut chargé des travaux de l'entretien et de la restauration de la cathédrale Saint-Julien du Mans. Son nom apparait dans le Nouveau dictionnaire biographique et critique des architectes français de Bauchal ${ }^{4}$, et une courte notice lui est consacrée dans le répertoire des architectes diocésains du XIX ${ }^{\mathrm{e}}$ siècle $^{5}$.

Un ouvrage récemment édité, venu enrichir une bibliographie peu abondante, souligne l'importance numérique et la remarquable variété de l'œuvre de PierreFélix Delarue, qu'elle soit publique ou privée, comme illustrant la diversité de la création architecturale du XIX ${ }^{\mathrm{e}}$ siècle $^{6}$. L'intérêt récemment porté à cet architecte peut s'expliquer par l'actualité patrimoniale et architecturale de la ville du Mans. Depuis quelques années, la cathédrale Saint-Julien du Mans fait l'objet de campagnes de restauration qui se situent tant en rupture qu'en continuité avec l'action de

1. Voir : Charles Gourlier, Léon-Marie-Dieudonné Biet, Edme-Jean-Louis Grillon, Eugène Tardieu, Choix d'édifices publics projetés et construits en France depuis le commencement du XIXe siècle, Paris, Louis Colas, 1825-1850, t. 1.

2. Base Mérimée : référence PA72000015.

3. Jean-Michel Leniaud, "Les architectes diocésains ", Monuments historiques, $\mathrm{n}^{\circ} 113$, Profession architecte en chef des monuments historiques, janv.-fév. 1981, Paris, Monuments historiques - CNMHS, 1981, p. 3-9.

4. Charles Bauchal, "Delarue", Nouveau dictionnaire biographique et critique des architectes français, Paris, André Daly, 1887, p. 657-658.

5. Ce répertoire est consultable sur le site internet de l'École nationale des chartes et dans : Jean-Michel Leniaud, Les Cathédrales au XIXe siècle : étude du service des édifices diocésains, Paris, Economica, 1993, 984 p.

6. Damien Castel, Pierre-Félix Delarue (1795-1873) architecte, Le Mans, Cabinet de Fromentières, 2013, $202 \mathrm{p}$. 
Delarue en qualité d'architecte "diocésain " ${ }^{7}$. L'un des derniers projets en date, après l'abandon de la restitution de la flèche et des clochetons de la tour $\operatorname{sud}^{8}$, concerne la restauration du programme sculpté du portail méridional de la cathédrale, cette restauration faisant suite à la modification, en 2008, du couronnement du porche surmontant le portail, réalisé en 1840-1841 par Delarue. La question du devenir du porche méridional de Saint-Julien du Mans dans la première moitié du XIX ${ }^{\mathrm{e}}$ siècle constitue une illustration du champ d'intervention d'un architecte diocésain dans le contexte des politiques patrimoniales et urbaines de son temps.

En l'état actuel des études disponibles sur Pierre-Félix Delarue, l'absence d'identification d'un fonds d'archives privées qui lui soit propre oriente le chercheur vers une documentation qui est de trois sortes : les écrits et plans qui se rattachent aux différentes administrations et particuliers ayant passé commande auprès de l'architecte, les édifices construits ou restaurés par Delarue, et la bibliographie consacrée à cet architecte.

\section{Itinéraire d'un architecte départemental}

Pierre-Félix Delarue naît à Thiais en 1795. Il est formé au métier d'architecte à Alençon par son oncle Jean-Baptiste Delarue (1744-1838), alors architecte départemental de l'Orne' . Il demeure à Alençon pendant toute la durée de son apprentissage du métier d'architecte, auquel Jean-Baptiste Delarue le formait en l'associant notamment à sa propre activité d'architecte départemental de l'Orne, jusqu'à ce que Pierre-Félix succède à son oncle en accédant à ce poste en $1818^{10}$.

À la même époque, le département voisin de la Sarthe connaissait une absence d'architecte départemental ${ }^{11}$. La responsabilité des services départementaux d'architecture relevant de l'initiative des préfets, il incombait à ces derniers de recruter un

7. L'adjectif diocésain est employé entre guillemets dans ce contexte, par opposition avec le corps des architectes diocésains créé en 1848, spécialement dévolu à la prise en charge des travaux menés sur les édifices diocésains, en particulier les cathédrales. L'architecte Jean-Baptiste Lassus succéda à Pierre-Félix Delarue en tant qu'architecte diocésain du Mans en 1849. Avant 1848, l'entretien des édifices diocésains était confié aux architectes départementaux. Source : Jean-Michel Leniaud, "Les architectes diocésains ", Monuments historiques, $\mathrm{n}^{\circ} 113$, Profession architecte en chef des monuments historiques, janvier-février 1981, Paris, Monuments historiques - CNMHS, 1981, p. 3-9.

8. "Saint-Julien oublie sa flèche et soigne son portail ", Ouest France, 2 mars 2011, sur le site http:// www.lemans.maville.com : site consulté en décembre 2016.

9. Damien Castel, Pierre-Félix Delarue (1795-1873) architecte, Le Mans, Cabinet de Fromentières, 2013, p. 3.

10. Id.

11. Dans son ouvrage sur Pierre-Théophile Segretain, Chantal Callais relève les difficultés de recrutement d'architectes dans les services départementaux d'architecture, en raison de la nature souvent ingrate et fastidieuse des tâches à accomplir. Source : Chantal Callais, À corps perdu, Pierre-Théophile Segretain architecte (1798-1864). Les architectes et la fonction publique d'État au XIX siècle, La Crèche, Geste éditions, 2010, p. 181. 
architecte de leur choix, dont la nomination était ensuite soumise pour approbation à leur ministre de tutelle. Dans le cas de la Sarthe, faute de candidats, c'était l'ingénieur en chef des Ponts et chaussées qui assurait tant bien que mal les missions d'architecte départemental, en cumulant les deux fonctions ${ }^{12}$. À plusieurs reprises, l'architecte et inspecteur des Bâtiments civils Jean-Antoine Alavoine fut envoyé en mission au Mans afin d'y seconder l'ingénieur en chef. Il établit des rapports à l'attention du Conseil des bâtiments civils sur l'état des chantiers en cours, à la cathédrale Saint-Julien notamment ${ }^{13}$, dont les travaux de restauration amorcés suite au rétablissement du culte consécutif au Concordat tardaient à être exécutés, et avaient même dû être interrompus en $1822^{14}$. Il y avait donc urgence à la cathédrale du Mans ${ }^{15}$, dont l'état général exigeait des réparations sans cesse ajournées.

Quand, le 25 janvier 1824, Pierre-Félix Delarue devient architecte départemental de la Sarthe ${ }^{16}$, il succède à l'ingénieur en chef Piou, démissionnaire en novembre $1823^{17}$. Le ministre de l'Intérieur rappelle à cette occasion que des instructions avaient été transmises au préfet de la Sarthe par le Conseil des bâtiments civils afin de s'occuper de la désignation d'un nouvel architecte départemental qui soit chargé des édifices diocésains ${ }^{18}$. Après sa nomination, Pierre-Félix Delarue cumule les fonctions d'architecte départemental de l'Orne et de la Sarthe jusqu'en 1830 environ $^{19}$.

12. Au cours de l'année 1818 , l'architecte et inspecteur des bâtiments civils Jean-Antoine Alavoine avait été invité à dresser des plans pour pallier les insuffisances des projets proposés par l'ingénieur en chef de la Sarthe. Source: Archives nationales, F ${ }^{13} 1922$.

13. Archives nationales, lettre du ministre de l'Intérieur, 16 mai 1823, $\mathrm{F}^{4} 1072$.

14. Alavoine expliqua dans un rapport que les fonctions de l'ingénieur «ne lui laissaient pas le temps de s'occuper des travaux d'architecture, que ce genre de travaux étaient étrangers à ses études et qu'en conséquence le meilleur moyen d'arriver à une prompte exécution serait d'en charger un artiste ayant les connaissances et le loisir nécessaires". Source : Archives nationales, rapport fait au Conseil des bâtiments civils par M. Gourlier, sur un rapport fait à son excellence le ministre de l'Intérieur par M. Alavoine sur les travaux de restauration de la cathédrale du Mans, $1^{\text {er }}$ mars 1823 , $\mathrm{F}^{19} 7732$.

15. En novembre 1810, une violente tempête avait entraîné l'écroulement d'une partie d'une des voûtes de la cathédrale. Source : Frédéric Pichon, Essai sur les travaux faits à la cathédrale du Mans pendant le XIX e siècle, Le Mans, Leguicheux-Gallienne, 1876, p. 12.

16. Archives départementales de la Sarthe, lettre du préfet de la Sarthe au ministre de l'Intérieur, 31 janvier $1824,4 \mathrm{~N} 1$.

17. Archives départementales de la Sarthe, remise du service départemental des bâtiments civils à PierreFélix Delarue, 5 janvier 1824, 4 N 1.

18. Archives nationales, lettre du ministre de l'Intérieur, 16 mai 1823, $\mathrm{F}^{4} 1072$.

19. Yves Roth, «Pierre-Félix Delarue, architecte (1795-1873) ", Que l'angélus sonne! Les églises du XIXe siècle dans l'Orne, exposition réalisée par la Direction des Archives départementales - Conservation des objets d'art, présentée à la Halle au blé d'Alençon du 23 décembre 2000 au 28 janvier 2001, Alençon, département de l'Orne/direction des archives départementales, 2000, p. 31-32. 


\section{Pierre-Félix Delarue au Mans : la restauration du couronnement du porche méridional de la cathédrale (1837-1841)}

Pierre-Félix Delarue s'attèle dès 1824 aux travaux de réparation de la cathédrale Saint-Julien, en particulier son porche méridional, dont l'état de dégradation avait été souligné à deux reprises dans des rapports établis avant $1810^{20}$. Ce porche, édifié dans les années $1180^{21}$, surmonte un portail réputé pour son programme sculpté ${ }^{22}$. Il fut ouvert du côté sud de la nef dans les années 1140, à la faveur de travaux entrepris par le chapitre cathédral du Mans suite à un incendie survenu en 1134 . En 1810 , la superstructure du porche méridional avait été rasée ${ }^{23}$, laissant place à une simple terrasse couverte de bitume, devenue insuffisante au cours des ans à protéger l'édifice d'infiltrations qui menaçaient peu à peu le décor sculpté du portail sud. À cette époque, la quasi totalité des murs de la cathédrale était masquée par des constructions qui venaient y prendre appui. Il s'agissait pour la plupart de simples échoppes, parfois de maisons plus importantes. Dans le cas du porche méridional, l'arc de sa face occidentale était entièrement obstrué par une maison qui formait cour avec d'autres constructions au niveau de l'angle du bras sud du transept (ill. 1).

\section{Un chantier peut en cacher un autre : les dégagements de la cathédrale Saint-Julien à la lumière de ses chantiers de restauration}

La nécessité de dégager les abords de la cathédrale s'impose comme le moyen de venir à bout des différents travaux de restauration, dans une optique de sauvegarde et de connaissance architecturale du monument ${ }^{24}$. Un rapport de 1830 sur la réparation des contreforts de la nef évoque les difficultés posées par les "édifices particuliers qui encombrent la cathédrale », et auxquelles Pierre-Félix Delarue

20. Archives départementales de la Sarthe, rapport des citoyens Jollivet, le Chêne et Mauchien, commissaires nommés pour constater les dégradations de l'église cathédrale du Mans, 14 ventôse an 12, $3 \mathrm{~V} 9$. Archives nationales, rapport sur l'état des réparations à la cathédrale du Mans par AntoinePierre Guyot, architecte du département de la Sarthe, 28 janvier 1809, F ${ }^{19} 7732$.

21. Médiathèque de l'architecture et du patrimoine, Le Mans, Sarthe, cathédrale Saint-Julien, étude préalable à la restauration du porche sud, annexe 2, notice historique, Christiane Schmuckle-Mollard ACMH, décembre 1995, 2002/004/0007.

22. Étienne Bouton, "Le programme sculpté du portail de la pierre au laict ", Pascal Mariette, Grégoire Faulin, Nicolas Gautier, La Cathédrale du Mans : du visible à l’invisible, Le Mans, La Reinette, 2015, p. 62.

23. Frédéric Pichon, Essai sur les travaux faits à la cathédrale du Mans pendant le XIX ${ }^{e}$ siècle, Le Mans, Leguicheux-Gallienne, 1876, p. 12.

24. Sur les dégagements des abords des cathédrales au XIX ${ }^{\mathrm{e}}$ siècle, voir : Jean-Michel Leniaud, Les Cathédrales au XIX siècle : étude du service des édifices diocésains, Paris, Economica, 1993, p. 441-458. 


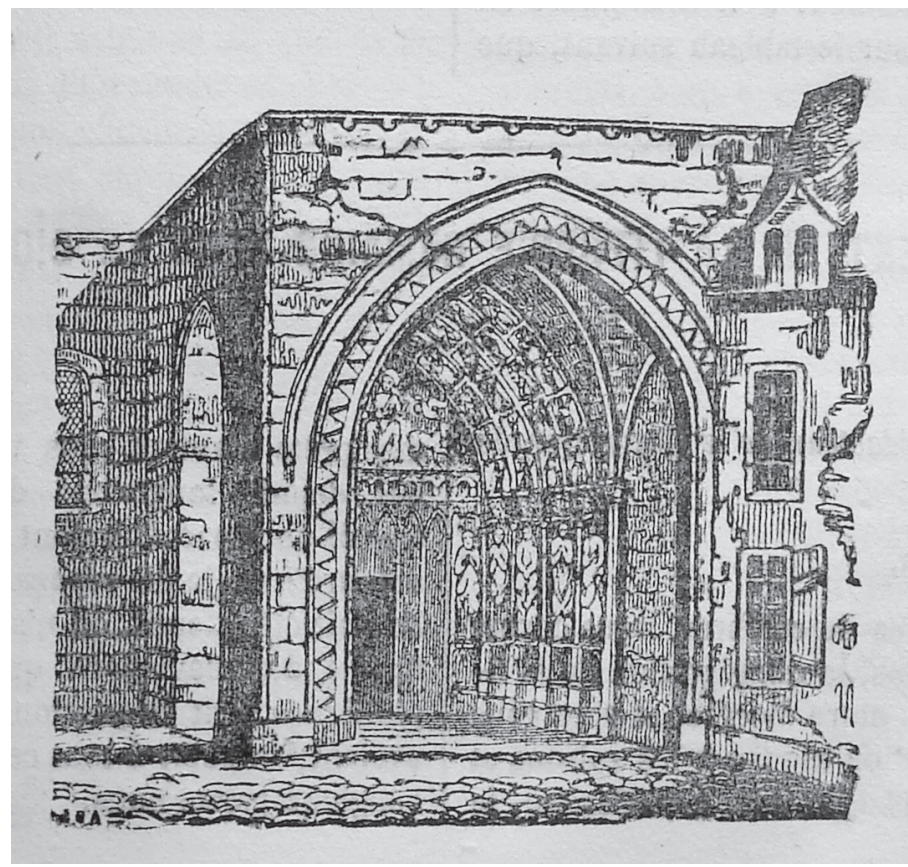

Ill. 1 : Vue du porche méridional, s.d. Source : La Chronique de l’Ouest, Le Mans, tome 1, 1856. (C) D. R.

remédie en établissant un système d'échafaudages adaptés aux contraintes environnementales du chantier ${ }^{25}$.

À cette dimension patrimoniale et technique des restaurations de Saint-Julien s'ajoute une dimension urbaine, dans la mesure où la municipalité tire parti des dégagements de la cathédrale pour effectuer des opérations de voirie, visant non seulement à désenclaver une partie de cet ancien quartier du Mans ${ }^{26}$, mais aussi à faciliter et embellir l'accès au porche méridional, ce à quoi l'évêque du Mans pouvait trouver intérêt ${ }^{27}$. Pierre-Félix Delarue intervient tant au niveau de la restauration du porche lui-même, que de l'aménagement urbain et la mise en valeur de ses abords.

25. Archives nationales, rapport sur la réparation des grands et petits contreforts au Conseil des bâtiments civils, Sarthe, Le Mans, cathédrale, ministère de l'Intérieur, séance du 13 avril 1830, $\mathrm{F}^{19} 7732$.

26. Robert Triger, Les Grandes transformations anciennes et modernes de la ville du Mans, Le Mans, Monnoyer, 1907, p. 46.

27. Médiathèque de l'architecture et du patrimoine, Le Mans, Sarthe, cathédrale Saint-Julien, dossiers généraux (1828-1886), lettre de l'évêque du Mans au ministre de la Justice et des Cultes, 10 mars 1837, 0080/041/0010. 


\section{Une opération d'urbanisme : le dégagement du porche méridional}

En 1837, un «très beau plan colorié » dressé par Delarue est présenté par l'abbé Lottin, secrétaire de l'évêché, au cours de la session générale annuelle de la Société française d'Archéologie ${ }^{28}$. Ce plan correspond à un projet d'isolement de la cathédrale, détaillé lors de la lecture d'un mémoire explicatif ${ }^{29}$. De ce projet d'isolement subsiste aujourd'hui une petite série de documents plus tardifs (aux alentours de $1843^{30}$ ), à savoir : un plan ${ }^{31}$, un projet de grille en fonte à placer autour de la cathédrale $^{32}$ et un rapport joint ${ }^{33}$. Ce projet servit de base au nouveau plan voyer du Mans pour les alignements des places Saint-Michel et du Château ${ }^{34}$. Il prévoit la mise en communication de l'ancienne cité avec la place des Jacobins au moyen d'un escalier ou d'une rue, question régulièrement posée au cours de l'histoire urbaine du Mans ${ }^{35}$. Pierre-Félix Delarue est également chargé de dresser les plans et procèsverbaux estimatifs des propriétés vouées à être acquises par l'État et démolies en vue du dégagement du porche. Ces documents apportent des informations sur des édifices aujourd'hui disparus du paysage manceau, dont quelques-uns ont récemment fait l'objet de prospections archéologiques et historiques ${ }^{36}$. Ces édifices furent acquis et démolis en $1837^{37}$, permettant à Pierre-Félix Delarue de prendre la pleine mesure de l'architecture du porche, et d'en proposer dès 1838 un projet de restauration.

\section{Les vicissitudes d'une restauration : entre archéologie et création}

Ce projet consiste en un plan et un devis détaillé contenant une introduction explicative $^{38}$. L'absence de couronnement au-dessus du porche depuis 1810 laisse le restaurateur face à un vide architectural qu'il importe de combler, mais comment? Delarue s'en remet à l'observation de la nef : «Pour cette reconstruction, autant qu'il

28. Société française d'archéologie, Bulletin monumental, Paris, Lance, 1837, t. 3, p. 319-320.

29. Archives historiques du diocèse du Mans, manuscrit, s.d., 3177.

30. Le dégagement complet de la cathédrale fut progressivement mené jusque dans les années 1850 , au gré des acquisitions successives des propriétés particulières.

31. Médiathèque de l'architecture et du patrimoine, 0080/041/0010.

32. Archives nationales, CP $\mathrm{F}^{19} 7733$.

33. Archives nationales, rapport sur l'isolement de la cathédrale du Mans par Delarue, 15 février 1843, $\mathrm{F}^{19} 7733$.

34. Archives départementales de la Sarthe, ville du Mans, plans d'alignements, rapport présenté au conseil municipal dans sa séance du 20 janvier 1847, par Tardif, auteur du nouveau plan voyer, 3 O 1029.

35. L'architecte Jean-Baptiste Lassus y répondit par la construction de l'actuel escalier du Jet-d'Eau. Source : Didier Travier, Les Jacobins, urbanisme et sociabilité au Mans, Le Mans, La Reinette, 2007, p. 31-35.

36. Hugo Meunier, "Le quartier canonial du Mans ", Pascal Mariette, Grégoire Faulin, Nicolas Gautier, La Cathédrale du Mans : du visible à l'invisible, Le Mans, La Reinette, 2015, p. 15-29.

37. Archives nationales, édifices diocésains, liquidation des dépenses, Le Mans, exercices 1838 et 1839 , $\mathrm{F}^{19} 4632$.

38. Archives départementales de la Sarthe, édifices diocésains, cathédrale du Mans, travaux extraordinaires, devis établis par Pierre-Félix Delarue, 1838, 3 V 12. 


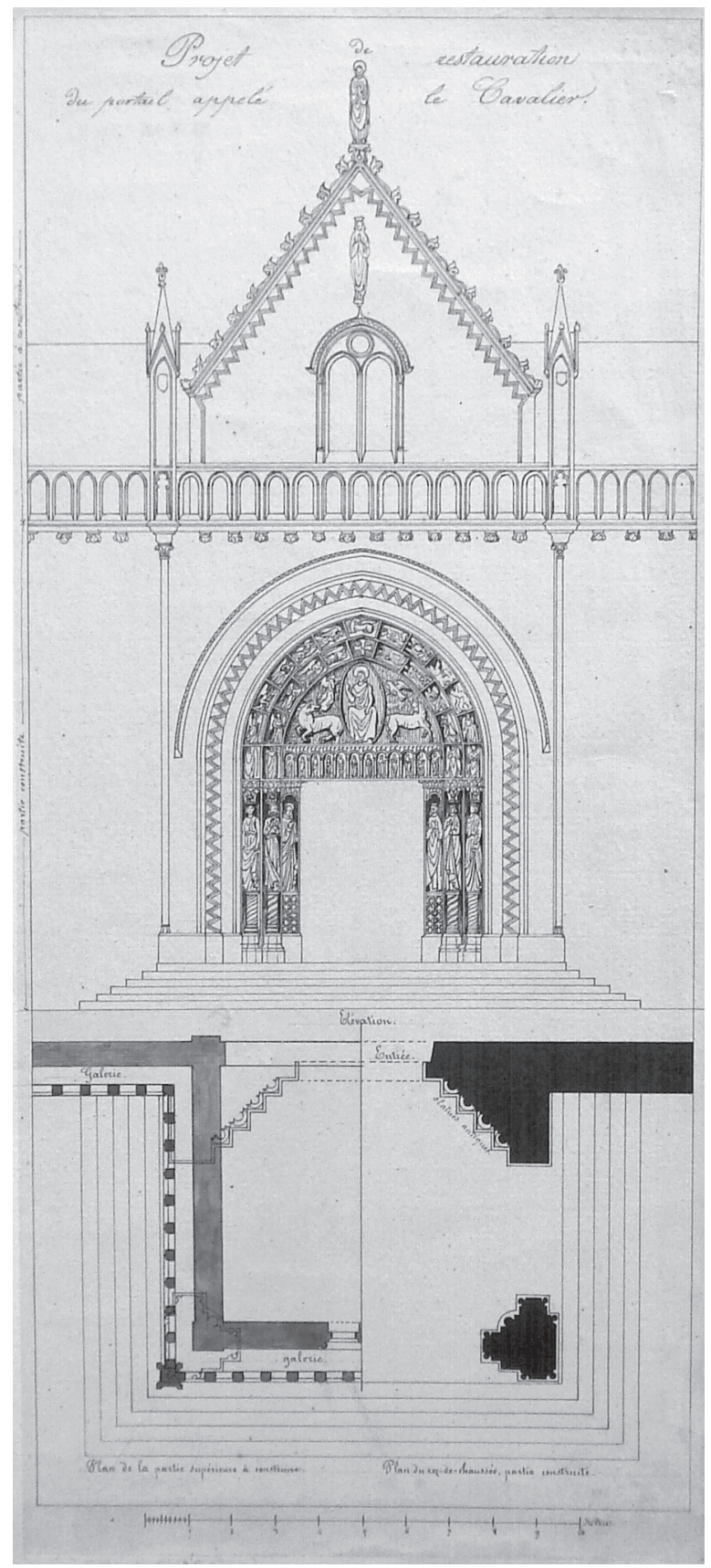

Ill. 2 : Projet de restauration du portail appelé le Cavalier, Pierre-Félix Delarue, 22 janvier 1838. (C) Médiathèque de l'architecture et du patrimoine. 
a été possible on a recherché quelle pouvait être l'ancienne disposition et pour la composition on s'est rattaché au profil des parties existantes ${ }^{39}$ ". Si l'on se fie au plan dessiné en 1838 (ill. 2), Pierre-Félix Delarue propose d'élever au-dessus du porche une balustrade sur ses trois faces, et un pignon aux rampants ornés d'éléments végétaux, encadré par des pyramidions, le tout semblant directement inspiré, en matière de "profil des parties existantes", des pinacles des arcs-boutants des hauts murs de la nef, construits en 1250-1270 ${ }^{40}$, et du pignon de la sacristie. Il semblerait que Delarue ait adopté la même démarche en 1843, au moment de dessiner la grille en fonte destinée à être placée tout autour de la cathédrale, dans le cadre du projet d'isolement du monument : "Cette grille sera établie par travée de 2 mètres [...] chaque travée sera placée entre des supports dont le style [...] est emprunté aux ornements choisis de la cathédrale ${ }^{41}$ ".

La restitution du couronnement du porche méridional n'est réalisée qu'en 1840-1841 ${ }^{42}$. Cependant ce couronnement, qui était encore visible en 2007, ne correspond en rien au plan dessiné par Delarue en 1838 (ill. 3). En lieu et place du pignon et de la balustrade prévus, figure un toit d'ardoises ceint par un crénelage de faible hauteur, suscitant de la part des historiens des commentaires aussi nombreux que variés. L'historien manceau Philippe Bouton en propose l'analyse suivante : « [...] de regrettables créneaux établis en 1839 ont voulu rappeler la tour élevée sur la porte du $\mathrm{XIV}^{\mathrm{e}}$ siècle, qui symbolisait en quelque sorte le pouvoir féodal du chapitre du Mans sur une partie de la ville. Elle avait été démolie en $1810^{43}$ ». Ambroise Ledru n'établit quant à lui aucun lien entre le crénelage mis en place par Delarue et l'existence antérieure de fortifications : "Je l'ai déjà dit, en 1830, l'architecte Delarue couronna le porche [...] d'un lourd toit crénelé, pour rappeler une fortification qui n'a d'ailleurs jamais existét ${ }^{4}$ ». Plus récemment, en 2015, Étienne Bouton explique qu'une tour crénelée avait bien été élevée au-dessus du porche au $\mathrm{XIV}^{\mathrm{e}}$ siècle, et qu'elle avait servi à loger les archives du chapitre de la cathédrale ${ }^{45}$.

Si rien à ce jour ne permet d'éclairer les raisons pour lesquelles le couronnement du porche ne fut pas restauré conformément au plan de 1838, la question de la documentation au XIX ${ }^{\mathrm{e}}$ siècle d'un état antérieur ayant pu servir de base à une restauration n'est pas sans réponse. Un dessin daté de $1695^{46}$ (ill. 4), représentant

39. Id.

40. Médiathèque de l'architecture et du patrimoine, Le Mans, Sarthe, cathédrale Saint-Julien, étude préalable à la restauration du porche sud, annexe 2, notice historique, Christiane SchmuckleMollard ACMH, décembre 1995, 2002/004/0007.

41. Archives nationales, rapport sur l'isolement de la cathédrale, Pierre-Félix Delarue, 15 février 1843, $\mathrm{F}^{19} 7733$.

42. Archives nationales, lettre du ministre de la Justice et des Cultes au préfet de la Sarthe, 30 septembre $1842, \mathrm{~F}^{19} 7733$.

43. Philippe Bouton, Le Vieux-Mans, richesses d'une ancienne cité, Angers, Philippe Petit, 1976, p. 34.

44. Ambroise Ledru, La Cathédrale du Mans Saint-Julien, Le Mans, E. Benderitter, 1923, p. 30.

45. Étienne Bouton, "Le programme sculpté du portail de la pierre au laict ", Pascal Mariette, Grégoire Faulin, Nicolas Gautier, op. cit., p. 62.

46. Bibliothèque nationale de France, département des estampes et de la photographie, L'Eglise St Iulien Cathédrale de la Ville du Mans, Veüe du costé du grand portail, 1695, Boudan Louis, Va-72 (3), MFILM H-149413. 


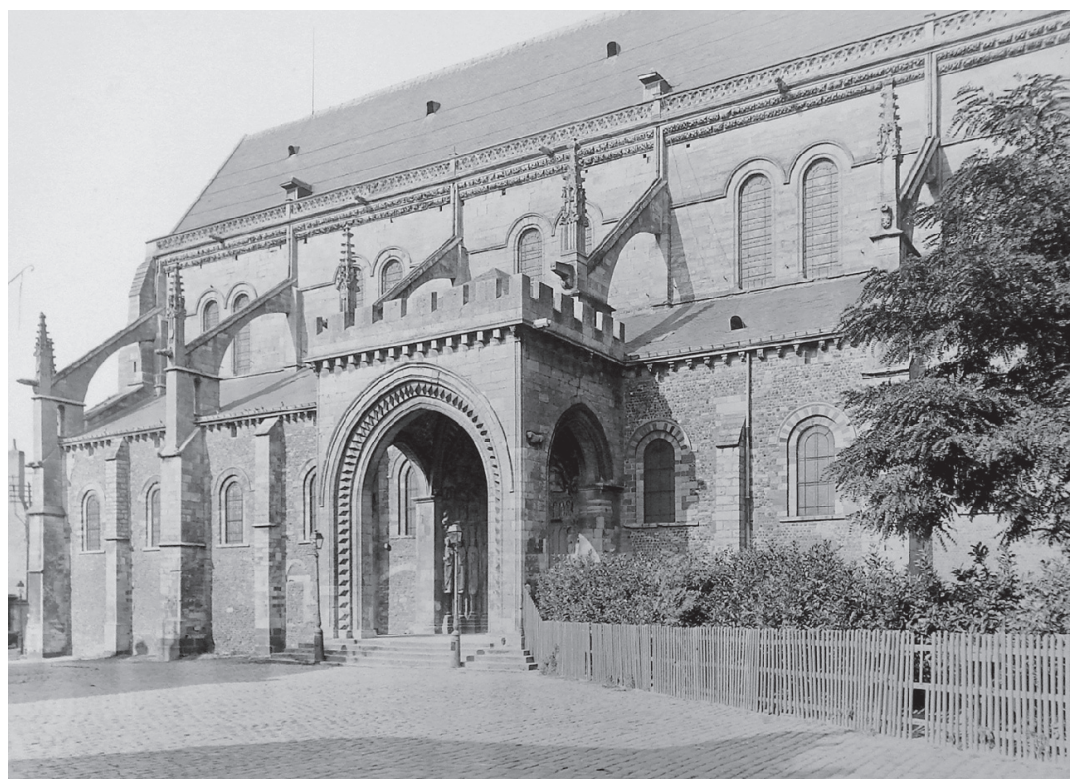

Ill. 3 : Cathédrale Saint-Julien, extérieur, nef sud, photographie. (C) Médiathèque de l'architecture et du patrimoine.

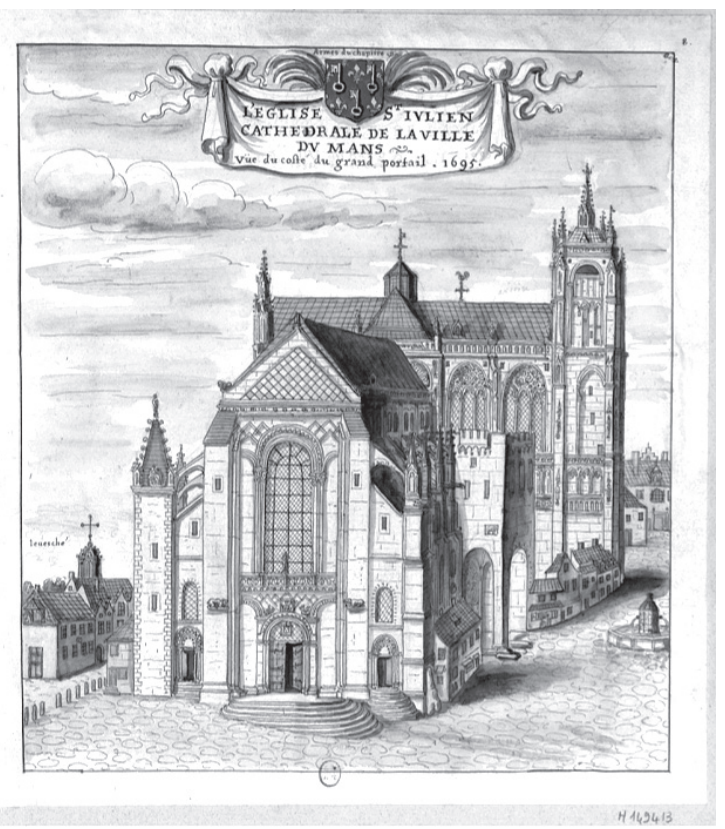

Ill. 4: L'Église St Iulien Cathédrale de la Ville du Mans, Veüe du costé du grand portail, 1695, Boudan Louis. (C) Bibliothèque nationale de France, Département des estampes et de la photographie. 
le porche méridional couronné par une tour élevée "vers le milieu du XIII siècle $^{47}$ ", fait actuellement partie des collections topographiques du département des estampes et de la photographie de la Bibliothèque nationale de France (de même qu'un autre dessin, représentant le porche méridional sous un aspect similaire $\left.{ }^{48}\right)$. Il n'est pas impossible qu'il ait pu faire partie au $\mathrm{XIX}^{\mathrm{e}}$ siècle des documents accessibles aux architectes de la cathédrale et aux historiens, puisque Jean-Baptiste Lassus en donne une description dans un rapport daté de $1851^{49}$. Entretemps, Viollet-le-Duc avait théorisé une méthode de travail à l'attention des architectes diocésains, qui rendait impossible toute modification d'un devis en cours de chantier, et en instituait le contrôle régulier ${ }^{50}$. Mentionnées à nouveau en $1988^{51}$ et 1995 dans une étude préalable $^{52}$, les dispositions anciennes du porche visibles sur ces deux dessins ne furent pas retenues en raison du coût de l'opération. Néanmoins, les découvertes de terrain et les recherches effectuées par les historiens, mentionnées par l'architecte en chef des monuments historiques dès 1988 , ont fourni des éléments de nature à réactualiser le débat sur la pertinence de la restauration de Delarue, et l'opportunité de maintenir un crénelage en mauvais état, qui est finalement déposé et remplacé par un toit en croupe en $2008^{53}$. La professionnalisation et l'institutionnalisation progressives de la protection du patrimoine architectural et de sa restauration à partir des années 1830, puis au moment du tournant de $1848^{54}$, furent globalement peu favorables à l'action de Delarue à Saint-Julien de 1824 à 1849. Son emploi de la fonte lui fut, à juste titre, particulièrement reproché5 ${ }^{55}$. L'on peut retenir de son

47. Médiathèque de l'architecture et du patrimoine, Le Mans, Sarthe, cathédrale Saint-Julien, étude préalable à la restauration du porche sud, Christiane Schmuckle-Mollard ACMH, décembre 1995, 2002/004/0007.

48. Bibliothèque nationale de France, département des estampes et de la photographie, Cathédrale du Mans, porche méridional, non daté, Va-72 (3), 149415.

49. "Les portefeuilles de la bibliothèque nationale contiennent plusieurs dessins provenant de la collection de Gaignières [...]. Ainsi l'un de ces dessins [...] représente d'ailleurs le porche latéral en perspective, et l'on distingue parfaitement la disposition des créneaux et mâchicoulis ». Source : médiathèque de l'architecture et du patrimoine, Charenton-le-Pont, rapport de Jean-Baptiste Lassus, 23 août 1851, 0080/041/0010.

50. Jean-Michel Leniaud, "Les architectes diocésains ", Monuments historiques, $\mathrm{n}^{\circ} 113$, Profession architecte en chef des monuments historiques, janvier-février 1981, Paris, Monuments historiques - CNMHS, 1981 , p. 3.

51. Direction régionale des affaires culturelles des Pays-de-la-Loire, Sarthe, cathédrale du Mans, projet de suppression du crénelage du porche sud, lettre de Hervé Baptiste ACMH, 9 juin 1988.

52. Médiathèque de l'architecture et du patrimoine, Le Mans, Sarthe, cathédrale Saint-Julien, étude préalable à la restauration du porche sud, Christiane Schmuckle-Mollard ACMH, décembre 1995, 2002/004/0007.

53. Médiathèque de l'architecture et du patrimoine, Le Mans, Sarthe, cathédrale Saint-Julien, restauration du porche royal, dossier documentaire des ouvrages exécutés, Marie-Suzanne de Ponthaud ACMH, mars 2008, 2010/003/0015.

54. Jean-Michel Leniaud, Les Cathédrales au XIXe siècle : étude du service des édifices diocésains, Paris, Economica, 1993, p. 26.

55. Archives nationales, rapport au ministre de l'Instruction publique et des Cultes sur l'état actuel de la cathédrale du Mans, 17 décembre 1849, $\mathrm{F}^{19} 7733$. 
long passage à la cathédrale du Mans la réalisation des relevés et calques des vitraux, la création d'un atelier pour leur restauration ${ }^{56}$, ainsi que la découverte des peintures des voûtes de la chapelle de la Vierge en $1842^{57}$. Le cas du couronnement du porche méridional illustre la complexité d'une opération de restauration monumentale, inscrite dans la dimension urbaine des "dégagements", entre "gros travaux ", " entretien ", "réparations ", et prémices de la connaissance archéologique et historique des édifices du Moyen Âge. Cette restauration a été remise en cause, non pas dans son principe, puisqu'elle se fondait sur la nécessité de dégager et de préserver l'ensemble du porche et du portail sculpté, mais dans son aspect. L'architecte restaurateur, en 1837 comme en 2007, pouvait en effet se trouver démuni face au problème posé par l'absence de couvrement au niveau du porche. Ce cas invitait à effectuer un travail de création spéculative, rejoignant en cela la définition de la restauration, devenue célèbre, proposée par Viollet-le-Duc dans son Dictionnaire raisonné de l'architecture ${ }^{58}$.

Delphine GOUTIERRE diplômée de premier cycle de l'École du Louvre, de l'université Paris I et de l'université du Maine

56. Actions qu'il ne manqua pas de rappeler au moment de son remplacement aux édifices diocésains par Jean-Baptiste Lassus en 1849. Source : Jean-Michel Leniaud, Jean-Baptiste Lassus, 1807-1857: ou le temps retrouvé des cathédrales, Paris, Arts et métiers graphiques, 1980, p. 75.

57. Sur fond de polémique au sujet de la technique à mettre en œuvre pour retirer les enduits qui recouvraient ces peintures. Source : Pascal Mariette, Grégoire Faulin, Nicolas Gautier, op. cit., p. 126

58. Eugène Viollet-le-Duc, Dictionnaire raisonné de l'architecture française du XI ${ }^{e}$ au XVI é siècle, Paris, Bance-Morel, 1854-1868, t. 8, "Restauration », p. 25-26, [en ligne]. 
